\title{
Eco-profiles für Kunststoffe
}

\section{Die Aussagekraft von Kennzahlen und Ökobilanzen hängt entscheidend von der Qualität der verfügbaren Informationen ab, auf denen sie aufbauen. Wie sehr diese Qualität zum einen vom jeweiligen ProduktionsprozeB, zum anderen aber auch vom Schutzinteresse des Unternehmens vor Datenabfluß bestimmt wird, zeigt der Beitrag aus der Sicht eines europäischen Kunststoffherstellers.}

D Von Michael Dröscher er Verband der Europäischen Kunststoffhersteller (APME, Association of Plastics. Manufacturers in Europe) hat zusammen mit Ian Boustead sogenannte „Eco-profiles“ für Standardkunststoffe herausgegeben (1-4). Ziel dieser Untersuchungen war es, ein Dateninventar zur Verfügung zu stellen, das die europäischen Gegebenheiten widerspiegelt. Diese internationalen Bilanzgrenzen sind richtig, denn Standardkunststoffe werden als commodities weltweit gehandelt. Zum Beispiel werden Kunststoffe für Verpackungen zu etwa 50 Prozent nach Deutschland importiert. Weiterhin gibt es Produzenten von Standardkunststoffen, die an bis zu zehn Standorten in Europa die gleichen Produkte herstellen.

Die Firma Hüls hat sich als Mitglied der APME an der Datenerfassung und Gestaltung dieser Eco-balance-Berichte beteiligt. Diese Arbeit ist nicht abgeschlossen, da seit 1992 ein Fortschritt in der Normungsaktivität zur Erhebung des Inventars von Ökobilanzen erzielt worden ist. Deshalb ist es auch erforderlich, die Berichte fortzuschreiben, was zur Zeit für den PolystyrolBericht auch geschieht.

Die Entscheidung, europäische Daten zu verwenden, setzt uns als Kunststoffhersteller in die Lage, unsere Datenbasis im Vergleich zum europäischen Mittel zu bewerten. Infolge der weit fortgeschrittenen Emissionsbegrenzung in Deutschland und der bereits sehr guten Energienutzung - so hat Hüls 1993 einen Energienutzungsgrad von mindestens 72 Prozent dank Kraft-Wärme-Kopplung erreicht - sind die Energie- und Rohstoffverbräuche sowie die Emissionsdaten der deutschen Hersteller oft günstiger als der europäische Schnitt. Deshalb stehen für einige Standardkunststoffe Datensätze auf Basis der Produktion in Deutschland zur Verfügung (5). Wie auch andere Hersteller veröffentlicht Hüls firmenspezifische Daten nicht, da es z.B. keinen Sinn macht, eine CD-Hülle im Markt auf eine einzelne Polystyrol-Produktionsanlage zurückzuführen. Dazu kommt, daß das Dateninventar immer nur die Situation zum Zeitpunkt der Datenerhebung widerspiegeln kann. Der Kunststoffherstellungsprozeß erstreckt sich beim Rohstoffhersteller vom Cracker bis hin zur Abpackung der Granulate oder bis zur Füllung des Silofahrzeuges mit Pulver.

\section{Begrenzte Aussagekraft der Datensätze}

Bei sich ändernden Crackerauslastungen, bei Änderung des Produktportfolios eines Standortes und damit verbundenen Änderungen der Energienutzung, bei Auslastungsschwankungen und natürlich bei verbesserter Technik, kommt es zu Abweichungen des aktuellen Dateninventars.

Bei all den Schwächen, die mit den Datensätzen verbunden sind, sind sie doch die einzige Basis für Ökobilanzvergleiche. Keine Werkstoffgruppe weist eine vergleichbare Datenkompatibilität auf. Dies zeigt die zur Zeit laufende Ökobilanzstudie zum Thema Frischwasser- und Abwasserrohrsysteme, die vom Verband der Chemischen Industrie zusammen mit der Keramikindustrie und den Kunststoffrohrherstellern durchgefuihrt wird, und die Studie des Umweltbundesamtes (UBA) zum Ökologievergleich von Polyvinylchlorid (PVC)-Bauprodukten.

Aus unseren Abnehmerbranchen, z.B. der Automobilindustrie, werden wir gelegentlich um Daten $\mathrm{zu}$ technischen Kunststoffen und anderen Werkstoffen gebeten, die nicht den Standardkunststoffen zuzurechnen sind. Der Aufwand, der mit der Erhebung dieser Daten verknüpft ist, ist in vielen Fällen nicht $\mathrm{zu}$ rechtfertigen, da bei vernetzten Produktionen schon allein die Frage der Allokation schwierig ist und einen großen Untersuchungsaufwand erfordert. Da diese Kunststoffprodukte im Endprodukt, z.B. einem Auto, mengenmäßig meist nur eine untergeordnete Menge ausmachen, wenn sie auch wichtige Funktionen erfillen, sollten hier eher Schätzungen als quantitative Daten verwendet werden. Schwierig ist es auch, wenn nur wenige Hersteller im Markt sind und die Daten den Einzelbeitrag direkt widerspiegeln. Hier hat Hüls dem UBA ausnahmsweise einen allerdings stark zusammengezogenen Datensatz zum Weichmacher Diethylhexylphthalat zur Verfügung gestellt. Summarische Daten sind zwar für den Wissenschaftler, der Ökobilanzen bearbeitet, nicht befriedigend, schützen aber den Datengeber vor Informationsabfluß.

\section{- Für vergleichende Ökobilanzen akzeptabel}

Zusammenfassend kann festgestellt werden, daß die von Boustead herausgegebenen Berichte mit allen genannten Einschränkungen eine akzeptable Datenbasis für vergleichende Ökobilanzen liefern. Ferner dienen sie dem einzelnen Hersteller als Meßlatte für seine Position im europäischen Wettbewerb. Ökobilanzvergleiche, die auf diesen Berichten beruhen, können allerdings nur Fallbeispiele abbilden, deren Übertragung in Allgemeinaussagen durch viele Randbedingungen begrenzt ist. Eine vollständige Bilanzierung, ausgehend vom Erdöl über den Cracker und eine spezifische Produktionsanlage bis hin zum fertigen Teil, vielleicht sogar bis zum fertigen Auto, kann nur wenig belastbare Durchschnittswerte liefern und stellt zudem eine Blitzlichtaufnahme des gegenwärtigen Standes der Technik dar.

\section{Literatur}

1) Eco-balance methodology for Commodity Thermoplastics, lan Boustead, a report for the European Centre for Plastics in the Environment, December 1992

2) Eco-profiles of the European plastics industry, Ian Boustead, reports for the European Centre for Plastics in the Environment, Reports: Nr. 2: Olefin Feedstock Sources, May 1993, Nr. 3: Polyethylene and Polypropylene, May 1993, Nr. 4: Polystyrene, May 1993

3) Eco-profiles of the European polymer industry, Ian Boustead, reports for APME's Technical and Environmental Centre, Reports: Nr. 5: Co-Product allocation in chlorine plants, April 1994, Nr. 6: Polyvinyl Chloride, April 1994, Nr. 7: Polyethylene Terephthalate (PET), April 1995

5) Die Datensätze können beim Verband Kunststofferzeugender Industrie, Karlstr. 21, 60329 Frankfurt

\section{Der Autor}

Michael Dröscher leitet bei Hüls die Abteilung Kunststoffe und Umwelt

Kontakt: Hüls Aktiengesellschaft, 45764 Marl, Tel. (02365) 49-2109, Fax -5405 
(c) 20I0 Authors; licensee IÖW and oekom verlag. This is an article distributed under the terms of the Creative Commons Attribution Non-Commercial No Derivates License (http://creativecommons.org/licenses/by-nc-nd/3.o/), which permits unrestricted use, distribution, and reproduction in any medium, provided the original work is properly cited. 